\title{
Editorial: Recent Advances in Biocatalysis: Focusing on Applications of These Processes
}

\author{
Immacolata Serra ${ }^{1 *}$, Lorena Wilson ${ }^{2}$ and Andrés R. Alcántara ${ }^{3}$ \\ ${ }^{1}$ Department of Biotechnology and Biosciences, University of Milano-Bicocca, Milan, Italy, ${ }^{2}$ Pontificia Universidad Católica de \\ Valparaíso, Valparaíso, Chile, ${ }^{3}$ Department of Chemistry in Pharmaceutical Sciences (QUICIFARM), Pharmacy Faculty, \\ Complutense University of Madrid (UCM), Madrid, Spain
}

Keywords: biocatalysis, biotransformations, enzymes, whole-cell biocatalysis, enzyme engineering, process optimization

Editorial on the Research Topic

Recent Advances in Biocatalysis: Focusing on Applications of These Processes

\section{OPEN ACCESS}

Edited and reviewed by: Georg M Guebitz,

University of Natural Resources and

Life Sciences Vienna, Austria

${ }^{*}$ Correspondence: Immacolata Serra

immacolata.serra@unimib.it

Specialty section:

This article was submitted to Industrial Biotechnology,

a section of the journal

Frontiers in Bioengineering and

Biotechnology

Received: 28 December 2021

Accepted: 24 January 2022

Published: 17 February 2022

Citation:

Serra I, Wilson L and Alcántara AR (2022) Editorial: Recent Advances in Biocatalysis: Focusing on Applications of These Processes.

Front. Bioeng. Biotechnol. 10:844741. doi: 10.3389/fbioe.2022.844741
Applied biocatalysis, that is, the use of biocatalysts (as whole cells or isolated enzymes, either in their natural state or chemically and/or genetically modified) is undoubtedly a very powerful tool for the practical implementation of sustainable industrial processes with maximum resource utilization and minimum waste generation, inside the context of a circular bio-based economy. In such a scenario, the Research Topic Recent Advances in Biocatalysis: Focusing on Applications of these Processes is presenting very exciting examples illustrating the broad applicability of biocatalysis.

Thus, Wang et al. reported how the combined use of a biocatalyzed step (whole cells from fungi) and an extraction of phenolic compounds is leading to an effective production of Iturin A (a cyclolipopeptide generally applied in the biological control of plant diseases) starting from rapeseed meal (RSM), a major by-product of oil extraction from rapeseed. This is a nice example illustrating the combination of biological pretreatment with chemical extraction and biotransformations from biobased residues to fine chemicals, according to the concept of biorefinery.

Capusoni et al. screened a collection of 28 yeasts isolated from different environments, marine and terrestrial, and identified new phytase activities in yeasts such as C. jadinii, K. marxianus, and T. delbrueckii. In particular, C. jadinii CJ2 was the best producer of both secreted and cell-bound phytase which showed a remarkable activity at high temperature and acidic $\mathrm{pH}$. These characteristics make the $C$. jadinii enzyme a promising candidate for feed/food-related processes for phytic acid degradation.

On the other hand, Menegatti and Žnidaršič-Plazl presented the development of a microbioreactor between two plates using an amine transaminase immobilized (together with the cofactor pyridoxal phosphate (PLP)) in a porous copolymeric hydrogel matrix. This experimental array did not require either organic solvents or any additional polycationic polymers for successful PLP retention, and allowed an enhanced enzymatic stability over a wider $\mathrm{pH}$ and temperature range compared to the free enzyme, as it was possible to retain $92 \%$ of the initial productivity after 10 days of continuous operation, even at $50^{\circ} \mathrm{C}$.

One of the current drawbacks of commercially available enzymes is derived from their mesophilic origin, which limits the optimal ranges of temperature and $\mathrm{pH}$ (i.e., between 20 and $45^{\circ} \mathrm{C}$, neutral $\mathrm{pH}$ ) that can be used. Thus, for industrial applications, an efficient solution is to use enzymes from extremophiles, which display higher activity, stability, and robustness compared to mesophilic counterparts, therefore allowing the development of biocatalysis at nonstandard conditions. In this sense, Espina et al. have reported a stepwise strategy for the development (cloning, over-expression, 
and downstream processing) and industrial production of three different extremozymes (a psychrotolerant catalase, a thermoalkaliphilic laccase, and a thermophilic aminetransaminase) isolated from several extreme environmental conditions in Antarctica, thus expanding the arsenal of available biocatalysts.

A different strategy to obtain biocatalysts with improved properties is represented by protein engineering. In this frame, Liu et al. described the identification of the key residues controlling the enantioselectivity of esterase Est924 belonging to the bSHL family and previously identified from a metagenomic library of bamboo root soil. In addition, rational engineering was applied to this enzyme, resulting in variants switching enantioselectivity (from $(R)$ to $(S)$ toward different ethyl 2arylpropionates), including some non-steroidal antiinflammatory drugs such as ketoprofen, naproxen, and ibuprofen. Finally, using whole-cell biocatalysts harboring one of the variants (M3, I202F/A203W/G208F), the kinetic resolution of ketoprofen and naproxen ethyl esters to the corresponding $(S)$ carboxylic acids was achieved with 95 and $96 \%$ ee, respectively, and with good biocatalyst recyclability.

A similar approach was used by Pan et al. to obtain a variant of a keratinase from Pseudomonas aeruginosa (KerPA) with increased activity and thermostability for industrial application. The best selected variant, Y21pBpF/Y70pBpF/ $\mathrm{Y} 114 \mathrm{pBpF}$, obtained through the insertion of non-canonical amino acids, showed, compared to the wild-type enzyme, an increased activity and half-life. This paper is an important example of how the incorporation of non-canonical amino acids into a protein may greatly expand the range of new possible interactions between the side chains of the residues and, consequently, promoting the creation of new molecular structures that result in enhanced physical, chemical, and biological properties of the mutated enzymes.

\section{AUTHOR CONTRIBUTIONS}

IS and ARA wrote the draft of the manuscript. LW revised the manuscript. All authors approved the final version of the submitted version.

Conflict of Interest: The authors declare that the research was conducted in the absence of any commercial or financial relationships that could be construed as a potential conflict of interest.

Publisher's Note: All claims expressed in this article are solely those of the authors and do not necessarily represent those of their affiliated organizations, or those of the publisher, the editors and the reviewers. Any product that may be evaluated in this article, or claim that may be made by its manufacturer, is not guaranteed or endorsed by the publisher.

Copyright $\odot 2022$ Serra, Wilson and Alcántara. This is an open-access article distributed under the terms of the Creative Commons Attribution License (CC BY). The use, distribution or reproduction in other forums is permitted, provided the original author(s) and the copyright owner(s) are credited and that the original publication in this journal is cited, in accordance with accepted academic practice. No use, distribution or reproduction is permitted which does not comply with these terms. 